\title{
A BROCARD-RAMANUJAN-TYPE EQUATION WITH LUCAS AND ASSOCIATED LUCAS SEQUENCES
}

\author{
István Pink AND MÁRTON SzIKSZAi \\ University of Salzburg, Austria and University of Debrecen, Hungary
}

\begin{abstract}
This paper deals with a Brocard-Ramanujan-type equation of the form

$$
u_{n_{1}} u_{n_{2}} \ldots u_{n_{k}}+1=u_{m}^{2}
$$

in unknown nonnegative integers $k, n_{1}, n_{2}, \ldots, n_{k}$ and $m$ with $k \geq 1$, where $u=\left(u_{n}\right)_{n=0}^{\infty}$ is either a Lucas sequence or its associated sequence. For certain infinite families of sequences we completely solve the above equation, extending some results of Marques [15], Szalay [21] and Pongsriiam [18]. The ingredients of the proofs are factorization properties of Lucas sequences, the celebrated result of Bilu, Hanrot and Voutier on primitive divisors of Lucas sequences and elementary estimations concerning the terms involved.
\end{abstract}

\section{INTRODUCTION}

Brocard $([4,5])$ and independently Ramanujan ([19]), unaware of Brocard's papers, posed the diophantine equation

$$
n !+1=m^{2}
$$

in positive integer unknowns $n$ and $m$. Solutions to (1.1) are called Brown numbers. Known Brown numbers are $(n, m)=(4,5),(5,11)$ and $(7,71)$, but it is still an open problem if there exists any other. Erdös conjectured that the above list of Brown numbers is complete, which is supported by considerable computational evidence. Namely, Gupta ([10]) showed that up to $n=63$ one cannot find an additional solution. This was later improved by Wells $([22])$ to

2010 Mathematics Subject Classification. 11B37, 11B39.

Key words and phrases. Brocard-Ramanujan equation, Lucas sequences.

The first author was granted by the Austrian science fund (FWF) under the project P 24801-N26. The second author was supported through the New National Excellence Program of the Ministry of Human Capacities of the State of Hungary. 
$n \leq 10^{7}$ and by a joint work of Berndt and Galway [2] to $n \leq 10^{9}$. Further, Overholt ([16]) proved that a weaker version of the $a b c$-conjecture implies the finiteness of Brown numbers, but his result does not give an effective upper bound on the sizes of the solutions.

Much study went into various generalizations of (1.1). Dąbrowski [6] verified finiteness for the setting

$$
n !+A=m^{2}
$$

with $A \in \mathbb{Z}$. However, his proof for square $A$ still assumes a weaker version of the $a b c$-conjecture. Another generalization was investigated by Berend and Osgood [1], who showed that if $P \in \mathbb{Z}[x]$ is of degree at least 2 , then the density of the set of positive integers $n$ such that

$$
P(x)=n !
$$

has a solution $x$, is zero. (1.2) was also considered by Erdős and Obláth ([9]) and Pollack and Shapiro ([17]) with $P(x)=x^{d} \pm 1$ and $d \geq 3$ being a prime. Provided that the full $a b c$-conjecture holds, Luca ([13]) found that (1.2) can have only finitely many solutions. We would like to point the interested reader to another paper of Dąbrowski [7], where additional generalizations are mentioned.

Variants of (1.1) involving linear recurrences have also been studied. Since the focus of this paper is the solution of equations of such type, we introduce some of the definitions at this point. Let $P$ and $Q$ be nonzero coprime integers such that the polynomial $x^{2}-P x+Q$ has two distinct roots $\alpha$ and $\beta$ with the property that $\alpha / \beta$ is not a root of unity. Define

$$
u_{n}=\frac{\alpha^{n}-\beta^{n}}{\alpha-\beta} \quad \text { and } \quad v_{n}=\alpha^{n}+\beta^{n} \quad(n \geq 0) .
$$

The sequences $u=\left(u_{n}\right)_{n=0}^{\infty}$ and $v=\left(v_{n}\right)_{n=0}^{\infty}$ are called the Lucas and associated Lucas sequences corresponding to the pair $(P, Q)$, respectively. Notable examples are the sequences of Fibonacci and Lucas numbers, both corresponding to the pair $(1,-1)$.

Luca and Shorey ([14]) investigated the equation

$$
u_{n} u_{n+1} \ldots u_{n+k-1}+t=y^{l},
$$

where $u=\left(u_{n}\right)_{n=0}^{\infty}$ is a Lucas sequence and $t$ is not a power of some rational number. They proved effective finiteness results on the solutions. A more specific equation has been considered by Marques ([15]), namely: he completely solved a Fibonacci analogue of (1.1), the equation

$$
F_{n} F_{n+1} \ldots F_{n+k-1}=F_{m}^{2}
$$

in positive integers $m, n, k$ with $k \geq 2$. In fact, he considered $k=1$ as well, but fails to find the sporadic solutions in that case. A more general version

$$
G_{n_{1}} G_{n_{2}} \ldots G_{n_{k}}+1=G_{m}^{2}
$$


was completely solved by Szalay $([21])$, where $G$ can be taken as the sequence of Fibonacci, Lucas or balancing numbers. We mention that Pongsriiam ([18]) extended the existing results for Fibonacci and Lucas numbers further by relaxing the conditions on the indices even more and investigating additional equations.

In this paper, we consider the equations

$$
u_{n_{1}} u_{n_{2}} \ldots u_{n_{k}}+1=u_{m}^{2}
$$

and

$$
v_{n_{1}} v_{n_{2}} \ldots v_{n_{k}}+1=v_{m}^{2}
$$

in unknown nonnegative integers $k, n_{1}, n_{2}, \ldots, n_{k}$ and $m$ with $k \geq 1, n_{1}<$ $n_{2}<\cdots<n_{k}$, where $u=\left(u_{n}\right)_{n=0}^{\infty}$ and $v=\left(v_{n}\right)_{n=0}^{\infty}$ are Lucas and associated Lucas sequences, respectively. We completely solve both (1.3) and (1.4) for the infinite families of sequences corresponding to the pairs of the form $(P, \pm 1)$ except a certain case for which the reason of exclusion will be discussed later. This way, we obtain an extension of the above mentioned results of Marques [15] and Szalay [21], respectively. Our solution is based on factorization properties of Lucas and associated Lucas sequences, the celebrated result of Bilu, Hanrot and Voutier [3] on primitive prime divisors and last, but not least, elementary estimations on the sizes of terms involved.

\section{NEW RESUlts}

Before formulating our results we make a quick note on "equivalent" solutions to (1.3) and (1.4). Let $\left(k, n_{1}, n_{2}, \ldots, n_{k}, m\right)$ be a solution. Observe that if $n$ is such that $n \neq n_{i}(i=1,2, \ldots, k)$ and $u_{n}=1$, then the inclusion of $n$ among the $n_{i}$-s yields a new solution, where $k$ is replaced by $k+1$. Obviously, the same can be said about the simultaneous inclusion of two terms with -1 values. Further, exclusion of terms from the solution in the same way is also possible. We call the solutions obtained by such constructions equivalent. Since \pm 1 values in Lucas and associated Lucas sequences can be listed explicitly without serious effort, see for instance, the papers of Hajdu and Szikszai $[11,12]$, and to avoid unnecessarily large number of solutions, we restrict ourselves to the representative of these equivalence classes, where $k$ is minimal.

Our first theorem concerns the complete solution of (1.3) for the pairs of the form $(P, \pm 1)$.

TheOREM 2.1. Let $u=\left(u_{n}\right)_{n=0}^{\infty}$ be the Lucas sequence corresponding to the pair $(P, \pm 1)$. Further, if $Q=-1$, then suppose that $P=1$ or that $m$ is 
odd. Then all solutions to equation (1.3) are given by

$$
\left(k, n_{1}, n_{2}, \ldots, n_{k}, m\right)= \begin{cases}(1,0,1) & \text { if } Q=1 \text { or } Q=-1 \\ (2, m-1, m+1, m) & \text { and } m \text { is odd }\end{cases}
$$

except the sequence corresponding to $(1,-1)$, where $\left(k, n_{1}, n_{2}, \ldots, n_{k}, m\right)=$ $(1,4,3),(1,6,4)$ and $(2, m-2, m+2, m)$ for even $m$ are also solutions.

In the second theorem, we turn our attention to (1.4) and find it has no solution for the pairs of the form $(P, \pm 1)$ apart from a few sporadic occurrences.

THEOREM 2.2. Let $v=\left(v_{n}\right)_{n=0}^{\infty}$ be the associated Lucas sequence corresponding to the pair $(P, \pm 1)$. Then (1.4) has no solution, except

$$
\left(k, n_{1}, n_{2}, \ldots, n_{k}, m\right)=(1,1,0)
$$

when $(P, Q)=(3, \pm 1)$ and

$$
\left(k, n_{1}, n_{2}, \ldots, n_{k}, m\right)=(1,2,0),(2,0,3,2)
$$

when $(P, Q)=(1,-1)$.

One may observe that in Theorem 2.2 , the case $Q=-1$ does not require any restrictions on $P$ or $m$. We discuss the reasons after the proofs are given.

In the following remark, we establish connection between a problem proposed in a paper of Dabrowksi and Ulas [8] and our results.

REMARK 2.3. The results of Theorem 2.1 and 2.2 yields a partial answer to Problem 4.10 in the extensive paper of Dąbrowski and Ulas [8]. There the authors ask for the description of the set of increasing functions $f: \mathbb{N}_{+} \rightarrow \mathbb{N}_{+}$ such that

$$
f(1) f(2) \ldots f(n)+1=f(m)^{2}
$$

has no solutions. Since the polynomials

$$
x^{2}-P x \pm 1
$$

have a dominant root, whenever $|P|>2$, we know that $u$ and $v$ are increasing at an exponential rate, thus falling under the scope of the above problem.

\section{Preliminary Results}

In what follows, we establish the background used in the proofs of our results. The first lemma concerns various factorization properties of Lucas and associated Lucas sequences.

LEMMA 3.1. Let $u=\left(u_{n}\right)_{n=0}^{\infty}$ and $v=\left(v_{n}\right)_{n=0}^{\infty}$ be the Lucas and associated Lucas sequences corresponding to the pair $(P, Q)$, respectively. The following properties hold. 
i) $v_{n}=u_{2 n} / u_{n}$;

ii) if $Q=1$, then $u_{n}^{2}-1=u_{n-1} u_{n+1}$;

iii) if $Q=-1$ and $n$ is odd, then $u_{n}^{2}-1=u_{n-1} u_{n+1}$;

iv) if $Q=1$ or $Q=-1$ and $n$ is even, then $v_{n}^{2}-1=u_{3 n} / u_{n}$.

Proof. All of the formulas can be obtained by using the defining relations of the sequences together with the Vieta's formula $Q=\alpha \beta$. We are not aware of any piece of literature that covers the whole statement in this form, however the book of Ribenboim [20] contains the relations scattered across Section 2.4.

If the factorization properties listed in Lemma 3.1 are applicable, both sides of our equations can be reduced to products involving terms of the sequences. The celebrated result of Bilu, Hanrot and Voutier [3] concerning primitive prime divisors is a powerful tool to compare the prime factors of these products. For the statement of the result we need the notion of primitive prime divisor. A prime $p$ is said to be a primitive prime divisor of the $u_{n}$ if $p \mid u_{n}$, but $p \nmid u_{m}$ for every positive $m<n$.

LEMma 3.2. Let $u=\left(u_{n}\right)_{n=0}^{\infty}$ be the Lucas sequence corresponding to the pair $(P, \pm 1)$. Then $u_{n}$ has a primitive prime divisor for every $n \geq 13$. Further, if $(P, Q) \neq(1,-1)$, then $u_{n}$ admit a primitive prime divisor for every $n \geq 5$.

Proof. In view of Theorem $\mathrm{C}$ in [3], it is enough to check if the pairs in Table 1 of [3] can yield any $(P, Q)$ with $Q= \pm 1$. Since this is not the case, the first part of the statement follows. The second part is implied by Theorem $\mathrm{D}$ and Table 3 there. Note that the definition of primitive prime divisor in [3] do not allow primes dividing $(\alpha-\beta)^{2}=P^{2}+4 Q$.

Note that we introduced Lucas and associated Lucas sequences as exponential functions of the roots of certain polynomials. However, these sequences are also binary linear recurrences. We use this fact in our proofs to solve (1.3) and (1.4) in several special cases.

LEMMA 3.3. Let $u=\left(u_{n}\right)_{n=0}^{\infty}$ and $v=\left(v_{n}\right)_{n=0}^{\infty}$ be the Lucas and associated Lucas sequences corresponding to the pair $(P, Q)$. Then for every $n \geq 0$ we have

$$
u_{n+2}=P u_{n+1}-Q u_{n} \quad \text { and } \quad v_{n+2}=P v_{n+1}-Q v_{n}
$$

with $u_{0}=0, u_{1}=1, v_{0}=2$ and $v_{1}=P$.

Proof. This is just an equivalent definition of the sequences.

Finally, we present a technical lemma needed to work out the case of (1.4) when $Q=-1, m$ is odd. There we do not have any useful factorization properties and need to rely on another approach. 
LEMMA 3.4. Let $2 \leq n_{1}<n_{2}<\cdots<n_{k}$ be positive integers with $k \geq 1$ and let $x>1$ be a positive real number. Consider the function

$$
f(x)=f_{n_{1}, n_{2}, \cdots, n_{k}}(x):=\left(1+\frac{1}{x^{2 n_{1}}}\right) \cdots\left(1+\frac{1}{x^{2 n_{k}}}\right) .
$$

Then

$$
f(x)=1+\xi
$$

where

$$
\frac{1}{x^{2 n_{1}}} \leq \xi<e^{\frac{1}{x^{2}\left(x^{2}-1\right)}}-1 .
$$

Further, for $x \geq 1+\sqrt{2}$ the representation (3.2) holds with

$$
\frac{1}{x^{2 n_{1}}} \leq \xi<0.037
$$

Proof. One can easily see from (3.1) that for any positive value $x>1$ the function $f(x)$ can be written in the form (3.2) with some $\xi>0$. Since $k \geq 1$, we have $\frac{1}{x^{2 n_{1}}} \leq \xi$ proving the lower bound in (3.3) (and (3.4)). For obtaining the upper bound in (3.3) we combine (3.2) with the inequality between the geometric and arithmetic means. Since

$$
\prod_{i=1}^{k}\left(1+\frac{1}{x^{2 n_{i}}}\right) \leq\left(\frac{k+\sum_{i=1}^{k} \frac{1}{x^{2 n_{i}}}}{k}\right)^{k}=\left(1+\frac{\sum_{i=1}^{k} \frac{1}{x^{2 n_{i}}}}{k}\right)^{k}
$$

we get

$$
1+\xi=f(x) \leq\left(1+\frac{\sum_{i=1}^{k} \frac{1}{x^{2 n_{i}}}}{k}\right)^{k}
$$

By assumption $2 \leq n_{1}<n_{2}<\cdots<n_{k}$ are integers and we have that $n_{i} \geq n_{1}+(i-1)$ for $1 \leq i \leq k$, whence by $x>1$ (and $n_{1} \geq 2$ ) we may write (3.6)

$$
\sum_{i=1}^{k} \frac{1}{x^{2 n_{i}}} \leq \frac{1}{x^{2 n_{1}}}\left(1+\frac{1}{x^{2}}+\cdots+\frac{1}{x^{2(k-1)}}\right)<\frac{x^{2} /\left(x^{2}-1\right)}{x^{2 n_{1}}} \leq \frac{1}{x^{2}\left(x^{2}-1\right)} .
$$

Now, the combination of (3.5) and (3.6) gives

$$
1+\xi \leq\left(1+\frac{\sum_{i=1}^{k} \frac{1}{x^{2 n}}}{k}\right)^{k}<e^{\frac{1}{x^{2}\left(x^{2}-1\right)}}
$$

which proves the upper bound (3.3). Finally, the upper bound in (3.4) is an easy consequence of the upper bound in (3.3) with $x \geq 1+\sqrt{2}$. 


\section{Proofs of the Theorems}

Proof of Theorem 2.1. Note that the choice $(P, Q)=( \pm 1,-1)$ gives the sequence of Fibonacci numbers or the Fibonacci numbers with alternating signs. In these cases, solutions can be obtained trivially from the results of Szalay [21]. Hence, without further mention, we suppose that $(P, Q) \neq$ $( \pm 1,-1)$.

Let $u=\left(u_{n}\right)_{n=0}^{\infty}$ be the Lucas sequence corresponding to the pair $(P, \pm 1)$. Substract 1 from both sides of (1.3) to get

$$
u_{n_{1}} u_{n_{2}} \ldots u_{n_{k}}=u_{m}^{2}-1
$$

Depending on $Q$ we apply either ii) or iii) of Lemma 3.1. This yields

$$
u_{n_{1}} u_{n_{2}} \ldots u_{n_{k}}=u_{m-1} u_{m+1} .
$$

First suppose that $m \geq 6$. From Lemma 3.2 it follows that both $u_{m-1}$ and $u_{m+1}$ admits a primitive prime divisor. Comparing the prime factors on both side of (4.1), a simple argument shows that $u_{n_{k}}=u_{m+1}$ and $u_{n_{k-1}}=u_{m-1}$. Thus $k=2$ and all the solutions in this case are given by

$$
\left(k, n_{1}, n_{2}, \ldots, n_{k}, m\right)=(2, m-1, m+1, m) .
$$

Now assume $m \leq 5$. Note that $n_{k} \leq 6$ also holds in this case. Otherwise, using Lemma 3.2 and a simple argument we show that there are no solutions. Observe that using Lemma 3.3 for a fixed value of $Q$ we can write $u_{n}$ as a polynomial in $P$. For instance, take $Q=-1$. Then the first few terms of $u$ are $0,1, P, P^{2}+1, P^{3}+2 P, \ldots$ Since $Q= \pm 1$ and we only have finitely many possibilities for $n_{1}, n_{2}, \ldots, n_{k}$ and $m$, we can check all of the corresponding polynomial equations and obtain the result.

Proof of Theorem 2.2. As in the proof of Theorem 2.1 we can exclude the case $(P, Q)=( \pm 1,-1)$, since it gives the sequence of Lucas numbers or the Lucas numbers with alternating signs, and those were also completely treated by Szalay [21].

Let $v=\left(v_{n}\right)_{n=0}^{\infty}$ be the associated Lucas sequence corresponding to the pair $(P, 1)$. Substract 1 from both sides of (1.4). We apply i) and iv) of Lemma 3.1 to get

$$
\frac{u_{2 n_{1}}}{u_{n_{1}}} \frac{u_{2 n_{2}}}{u_{n_{2}}} \cdots \frac{u_{2 n_{k}}}{u_{n_{k}}}=\frac{u_{3 m}}{u_{m}} .
$$

If $m \leq 4$, then $2 n_{k} \leq 12$, otherwise, by Lemma 3.2 , we would have that $u_{2 n_{k}}$ admit a factor that does not divide the right hand side of (4.2). Using Lemma 3.3 we write the terms of $v$ as polynomials in $P$. The first several terms are $2, P, P^{2}-2, P^{3}-3 P \ldots$ Using this we can reduce (1.4) to a polynomial equation and with $n_{1}, n_{2}, \ldots, n_{k}$ and $m$ bounded we check all of the possibilities. 
It remains to consider $m \geq 5$. By Lemma 3.2, both $u_{m}$ and $u_{3 m}$ admit a primitive prime divisor and a simple argument shows that $3 m=2 n_{k}$. First, assume that $k=1$. Then (4.2) becomes

$$
\frac{u_{2 n_{1}}}{u_{n_{1}}}=\frac{u_{3 m}}{u_{m}}
$$

Eliminate $u_{2 n_{1}}=u_{3 m}$ to get $u_{m}=u_{n_{1}}$. Now Lemma 3.2 implies $m=n_{1}$, contradicting $3 m=2 n_{1}$.

Take $k=2$. By rearranging the terms in (4.2) the equation simplifies to

$$
u_{2 n_{1}} u_{m}=u_{n_{2}} u_{n_{1}} .
$$

Since $u_{m}$ has a primitive divisor, we can get $m=n_{2}$ and $m=n_{1}$. The former is not possible, thus $m=n_{1}$ and we divide both sides to get

$$
u_{2 n_{1}}=u_{n_{2}} .
$$

Observe that $n_{2}=\frac{3}{2} m>5$ and hence $u_{n_{2}}$ has a primitive prime divisor. This yields $2 n_{1}=n_{2}$. Now we have the relations $3 m=2 n_{2}, 2 n_{2}=4 n_{1}$ and $4 n_{1}=4 m$, leading to a contradiction.

Finally, assume that $k \geq 3$. We rearrange the terms and eliminate $u_{3 m}=$ $u_{2 n_{k}}$ to get

$$
u_{2 n_{1}} u_{2 n_{2}} \ldots u_{2 n_{k-1}} u_{m}=u_{n_{1}} u_{n_{2}} \ldots u_{n_{k}} .
$$

Like before, $m=n_{k}$ is not a possibility. But $u_{n_{k}}$ has a primitive divisor, and we find that $n_{k}=2 n_{k-1}$. Now $3 m=2 n_{k}=4 n_{k-1}$ and hence $n_{k-2}<n_{k-1}=$ $\frac{3}{4} m$. We know that $u_{m}$ has a primitive divisor implying $m=n_{k-1}$. Once more, we find a contradiction, finishing the proof for $Q=1$.

When $Q=-1$ and $m$ is even we can verify the statement in an analogous way. However, when $m$ is odd and $Q=-1$ we do not have any useful factorization properties and hence prove it differently.

Consider equation (1.4) in positive integer unknowns $\left(n_{1}, n_{2}, \cdots, n_{k}, m\right)$ with $1 \leq n_{1}<n_{2}<\cdots<n_{k}$ and $m \geq 1$ odd. If among the numbers $1 \leq n_{1}<n_{2}<\cdots<n_{k}$ there exists at least one $n_{i}$ which is odd then $P=v_{1} \mid v_{n_{i}}$ and since $m$ is also odd we have that $P=v_{1} \mid v_{m}$ also holds. Hence equation (1.4) implies $P \mid 1$, which is, by $|P| \geq 2$ a contradiction. Thus in what follows, we may suppose that all the numbers $n_{i}(1 \leq i \leq k)$ are even and hence we may also assume that $n_{1} \geq 2$. Since $v_{j}=\alpha^{j}+\beta^{j}, \alpha \beta=-1, m$ is odd, $\alpha$ is a real number and each $n_{i}(1 \leq i \leq k)$ is even, equation (1.4) can be rewritten as

$$
|\alpha|^{t}\left(1+\frac{1}{|\alpha|^{2 n_{1}}}\right) \cdots\left(1+\frac{1}{|\alpha|^{2 n_{k}}}\right)=|\alpha|^{2 m}+\frac{1}{|\alpha|^{2 m}}-3 .
$$

where $t=n_{1}+n_{2}+\cdots+n_{k}$. By dividing both sides of (4.3) by $|\alpha|^{t}$ and using Lemma 3.4 with $x=|\alpha| \geq 1+\sqrt{2}$ we get that

$$
\frac{3}{|\alpha|^{t}}+1+\xi=|\alpha|^{2 m-t}+\frac{1}{|\alpha|^{2 m+t}} \text {. }
$$


where $\xi$ satisfies (3.4), that is $\frac{1}{x^{2 n} 1} \leq \xi<0.037$.

We distinguish three subcases according to $2 m=t, 2 m<t$ and $2 m>t$, respectively.

CASE I: $2 m=t$. If $2 m=t$, then $|\alpha|^{2 m-t}=1$ and hence (4.4) leads to

$$
\frac{3}{|\alpha|^{2 m}}+\xi=\frac{1}{|\alpha|^{4 m}},
$$

which, by $\xi>0, m \geq 1$ and $|\alpha| \geq 1+\sqrt{2}$, leads to a contradiction.

CASE II: $2 m<t$. If $2 m<t$, then, since $2 m$ and $t$ are integers, it follows that $2 m-t \leq-1$ and hence $|\alpha|^{2 m-t} \leq \frac{1}{|\alpha|}$. We have $m \geq 1$ and $t \geq n_{1} \geq 2$ implying $\frac{1}{|\alpha|^{2 m+t}} \leq \frac{1}{|\alpha|^{4}}$. Using these and the facts that $\frac{3}{|\alpha|^{t}}>0$ and $\xi>0$, equation (4.4) leads to

$$
1<\frac{1}{|\alpha|}+\frac{1}{|\alpha|^{4}}
$$

which is a contradiction in view of $|\alpha| \geq 1+\sqrt{2}$.

CASE III: $2 m>t$. If $2 m>t$, then, since $2 m$ and $t$ are integers, it follows that $2 m-t \geq 1$ and hence $|\alpha|^{2 m-t} \geq|\alpha|$. By $t \geq n_{1} \geq 2$ it implies $\frac{3}{|\alpha|^{t}} \leq \frac{3}{|\alpha|^{2}}$. Further, by (3.4) of Lemma 3.4 we know that $\xi>0$ is a positive number with $\xi<0.037$. Putting the above informations together and observing that $\frac{1}{|\alpha|^{2 m+t}}>0$ we see that (4.4) leads to

$$
|\alpha|<1+\xi+\frac{3}{|\alpha|^{t}} \leq 1.037+\frac{3}{|\alpha|^{2}},
$$

which is a contradiction again in view of $|\alpha| \geq 1+\sqrt{2}$. This finishes the proof.

We finish the paper by some notes. The first one concerns the differences in the assumptions of the theorems.

Remark 4.1. One may observe that Lemma 3.4 could have been extended in a way to solve the case excluded by the conditions of Theorem 2.1, but not by those of Theorem 2.2. However, the main difference show up in the application of such a technical lemma. In fact, the authors are not sure if there is an elementary way in which one can solve this remaining equation, at least as easily as for associated Lucas sequences. Since one of our goals is to keep the integrity of the paper by not considering anything else than certain divisibility properties and trivial estimates, we omit it. Neverthless, the interested reader is encouraged to solve this "elusive" equation either by disproving our intuition our applying deeper methods.

In what follows, we mention two possible generalizations.

Remark 4.2. In the paper of Bilu, Hanrot and Voutier [3], there are analogous results for Lehmer sequences as for Lucas sequences. Since factorization properties are very similar, one can expect a similar way to solve (1.3) 
and (1.4) with Lehmer sequences instead of Lucas sequences. However, the authors predict that either a different proof or a considerable modification of the last part of the proof of Theorem 2.2 is needed.

Remark 4.3. As it was mentioned in the Introduction, Pongsriiam ([18]) made further improvements on the results of Marques [15] and Szalay [21] by relaxing the conditions on the indices further. Namely, he allows the equality of the $n_{i}$-s in (1.3) and (1.4) and replaces +1 by \pm 1 . The authors expect that the ideas presented in the proofs of Theorem 2.1 and 2.2 can be applied there as well to obtain complete solution for pairs of the form $(P, \pm 1)$.

In view of Remark 4.2 and 4.3, we formulate the following problems.

Problem 4.1. Under the same assumptions as in Theorem 2.1 and 2.2, completely solve equation (1.3) and (1.4), when $u$ and $v$ are replaced by Lehmer and associated Lehmer sequences, respectively.

Problem 4.2. Under the same assumptions as in Theorem 2.1 and 2.2, completely solve the equations

$$
u_{n_{1}}^{\alpha_{1}} u_{n_{2}}^{\alpha_{2}} \ldots u_{n_{k}}^{\alpha_{k}} \pm 1=u_{m}^{2}
$$

and

$$
v_{n_{1}}^{\alpha_{1}} v_{n_{2}}^{\alpha_{2}} \ldots v_{n_{k}}^{\alpha_{k}} \pm 1=v_{m}^{2}
$$

to extend the results of Pongsriiam [18].

\section{ACKNOWLEDGEMENTS.}

The authors would like to thank the anonymous referee for his/her useful comments and for drawing our attention towards the papers $[7,18]$. The authors also thank Carlo Sanna for his careful reading of the draft and for his remarks which helped us improving it.

\section{REFERENCES}

[1] D. Berend and C. Osgood, On the equation $P(x)=n$ ! and a question of Erdös, J. Number Theory 42 (1992), 189-193.

[2] B. C. Berndt and W. F. Galway, On the Brocard-Ramanujan Diophantine equation $n !+1=m^{2}$, Ramanujan J. 4 (2000), 41-42.

[3] Y. Bilu, G. Hanrot and P. Voutier, Existence of primitive divisors of Lucas and Lehmer numbers (with an appendix by M. Mignotte), J. Reine Angew. Math. 539 (2001), 75122 .

[4] H. Brocard, Question 166, Nouv. Corresp. Math. 2 (1876), 287.

[5] H. Brocard, Question 1532, Nouv. Ann. Math. 4 (1885), 391.

[6] A. Dąbrowski, On the Diophantine Equation $x !+A=y^{2}$, Nieuw Arch. Wisk. 14 (1996), 321-324

[7] A. Dąbrowski, On the Brocard-Ramanujan problem and generalizations, Colloq. Math. 126 (2012), 105-110.

[8] A. Dąbrowski and M. Ulas, Variations on the Brocard-Ramanujan equation, J. Number Theory 133 (2013), 1168-1185. 
[9] P. Erdős and R. Obláth, Über diophantische Gleichungen der Form $n !=x^{p} \pm y^{p}$ und $n ! \pm m !=x^{p}$, Acta Litt. Sci. Szeged 8 (1937), 241-255.

[10] H. Gupta, On a Brocard-Ramanujan Problem, Math. Student 3 (1935), 71.

[11] L. Hajdu and M. Szikszai, On the GCD-s of $k$ consecutive terms of Lucas sequences, J. Number Theory 132 (2012), 3056-3069.

[12] L. Hajdu and M. Szikszai, Common factors in series of consecutive terms of associated Lucas and Lehmer sequences, Fibonacci Quart. 53 (2015), 221-229.

[13] F. Luca, The Diophantine equation $P(x)=n$ ! and a result of $M$. Overholt, Glas. Mat. Ser. III 37 (2002), 269-273.

[14] F. Luca and T. N. Shorey, Diophantine equations with products of consecutive terms in Lucas sequences II, Acta Arith. 133 (2008), 53-71.

[15] D. Marques, The Fibonacci version of the Brocard-Ramanujan Diophantine equation, Port. Math. 68 (2011), 185-189.

[16] M. Overholt, The Diophantine Equation $n !+1=m^{2}$, Bull. Lond. Math. Soc. 25 (1993), 104.

[17] R. M. Pollack and H. N. Shapiro, The next to last case of a factorial diophantine equation, Comm. Pure Appl. Math. 26 (1973), 313-325.

[18] P. Pongsriiam, Fibonacci and Lucas numbers associated with Brocard-Ramanujan equation, preprint. arXiv:1509.07898v1.

[19] S. Ramanujan, Question 469, J. Indian Math. Soc. 5 (1913), 59.

[20] P. Ribenboim, Little book of bigger primes, Second edition, Springer-Verlag, New York, 2004.

[21] L. Szalay, Diophantine equations with binary recurrences associated to the BrocardRamanujan problem, Port. Math. 69 (2012), 213-220.

[22] D. Wells, The Penguin Dictionary of Curious and Interesting Numbers, Penguin Books, Middlesex, England, 1986.

\section{Pink}

Institute of Mathematics

University of Debrecen

P.O. Box 12, H-4010 Debrecen, Hungary

and

University of Salzburg

Hellbrunnerstrasse 34/I, A-5020 Salzburg, Austria

E-mail: pinki@science.unideb.hu \& istvan.pink@sbg.ac.at

M. Szikszai

Institute of Mathematics

University of Debrecen

P.O. Box 12, H-4010 Debrecen, Hungary

E-mail: szikszai.marton@science.unideb.hu

Received: 3.6.2016.

Revised: 8.7.2016. \& 18.7.2016. 